\title{
Quantum Kinetic Equations of Many-Particle Systems in Condensed States
}

\author{
V.I. Gerasimenko*11 and Zh.A. Tsvir**2 \\ * Institute of Mathematics of NAS of Ukraine, \\ 3, Tereshchenkivs'ka Str., \\ 01601 Kyiv-4, Ukraine \\ ** Taras Shevchenko National University of Kyiv, \\ Department of Mechanics and Mathematics, \\ 2, Academician Glushkov Av., \\ 03187 Kyiv, Ukraine
}

\begin{abstract}
This paper is devoted to the description of the evolution of states of quantum many-particle systems within the framework of a one-particle density operator, which enables to construct the kinetic equations in scaling limits in the presence of correlations of particle states at initial time, for instance, correlations characterizing the condensed states.
\end{abstract}

Key words: quantum kinetic equation; nonlinear Schrödinger equation; scaling limit; condensed state; quantum many-particle system.

2000 Mathematics Subject Classification: 35Q40; 47J35; 47H20; 82C10; 82C22.

\footnotetext{
${ }^{1}$ E-mail: gerasym@imath.kiev.ua

22E-mail: Zhanna.Tsvir@simcorp.com
} 


\section{Contents}

1 Introduction $\quad 2$

2 The generalized quantum kinetic equation in case of correlated initial state 4

$\begin{array}{llr}3 & \text { The mean field limit theorems } & 7\end{array}$

4 On mean field quantum kinetic equations 8

5 Conclusion $\quad 9$

\begin{tabular}{ll} 
References & 9 \\
\hline
\end{tabular}

\section{Introduction}

Recently the considerable advance in the rigorous derivation of quantum kinetic equations in the mean field scaling limit [1], such as the nonlinear Schrödinger equation and the GrossPitaevskii equation [2 11] is observed. In the scaling limit approach to the construction of kinetic equations one of the essential assumptions is that initial data has to satisfy a chaos property, i.e. the initial state is completely determined by a sequence of products of the oneparticle density operators [1], [12. At the same time it is well known that, for instance, the equilibrium state of the Bose condensate is characterized by correlations of particles in contrast to the gaseous state [13. Thus, the validation of quantum kinetic equations in case of presence of correlations at initial time, in particular for condensed states, remains an actual problem so far.

In the paper we consider the problem of potentialities inherent in the description of the evolution of states of many-particle systems in terms of a one-particle density operator in case of presence of correlations of particles at initial time. We prove that, if initial data is completely specified by a one-particle marginal density operator and initial correlations, then all possible states of infinite-particle systems at arbitrary moment of time can be described without any approximations within the framework of a one-particle density operator governed by the kinetic equation, which coefficients are determined by the initial correlations. Then the actual kinetic equations that describe the evolution of interacting particles in the condensed states, are derived in appropriate scaling limits on the basis of the established quantum kinetic equation.

We consider a quantum system of a non-fixed (nonequilibrium grand canonical ensemble) number of identical (spinless) particles obeying Maxwell-Boltzmann statistics in the space $\mathbb{R}^{3}$. We will use units where $h=2 \pi \hbar=1$ is a Planck constant, and $m=1$ is the mass of particles.

Let $\mathcal{H}$ be a one-particle Hilbert space, then the $n$-particle space $\mathcal{H}_{n}$, is a tensor product of $n$ Hilbert spaces $\mathcal{H}$. The Hamiltonian $H_{n}$ of $n$-particle system is a self-adjoint operator with 
domain $\mathcal{D}\left(H_{n}\right) \subset \mathcal{H}_{n}$ :

$$
H_{n}=\sum_{i=1}^{n} K(i)+\epsilon \sum_{i_{1}<i_{2}=1}^{n} \Phi\left(i_{1}, i_{2}\right)
$$

where $K(i)$ is the operator of a kinetic energy of the $i$ th particle, $\Phi\left(i_{1}, i_{2}\right)$ is the operator of a two-body interaction potential and $\epsilon>0$ is a scaling parameter. The operator $K(i)$ acts on functions $\psi_{n}$, that belong to the subspace $L_{0}^{2}\left(\mathbb{R}^{3 n}\right) \subset \mathcal{D}\left(H_{n}\right) \subset L^{2}\left(\mathbb{R}^{3 n}\right)$ of infinitely differentiable functions with compact supports according to the formula: $K(i) \psi_{n}=-\frac{1}{2} \Delta_{q_{i}} \psi_{n}$. Correspondingly we have: $\Phi\left(i_{1}, i_{2}\right) \psi_{n}=\Phi\left(q_{i_{1}}, q_{i_{2}}\right) \psi_{n}$, and we assume that the function $\Phi\left(q_{i_{1}}, q_{i_{2}}\right)$ is translation-invariant bounded function which is symmetric with respect to permutations of arguments.

Let $\mathfrak{L}^{1}\left(\mathcal{H}_{n}\right)$ be the space of trace class operators $f_{n} \equiv f_{n}(1, \ldots, n) \in \mathfrak{L}^{1}\left(\mathcal{H}_{n}\right)$ that satisfy the symmetry condition: $f_{n}(1, \ldots, n)=f_{n}\left(i_{1}, \ldots, i_{n}\right)$ for arbitrary $\left(i_{1}, \ldots, i_{n}\right) \in(1, \ldots, n)$, and equipped with the norm: $\left\|f_{n}\right\|_{\mathfrak{L}^{1}\left(\mathcal{H}_{n}\right)}=\operatorname{Tr}_{1, \ldots, n}\left|f_{n}(1, \ldots, n)\right|$, where $\operatorname{Tr}_{1, \ldots, n}$ are partial traces over $1, \ldots, n$ particles. We denote by $\mathfrak{L}_{0}^{1}\left(\mathcal{H}_{n}\right)$ the everywhere dense set of finite sequences of degenerate operators with infinitely differentiable kernels with compact supports. Let $\mathfrak{L}\left(\mathcal{H}_{n}\right)$ be the space of bounded operators defined on the Hilbert space $\mathcal{H}_{n}$.

On the space $\mathfrak{L}^{1}\left(\mathcal{F}_{\mathcal{H}}\right)$ the one-parameter family of operators

$$
\mathcal{G}_{n}(-t) f_{n} \doteq e^{-i t H_{n}} f_{n} e^{i t H_{n}} .
$$

is defined and it is an isometric strongly continuous group which preserves positivity and selfadjointness of operators. For $f \in \mathfrak{L}_{0}^{1}\left(\mathcal{H}_{n}\right)$ there exists the infinitesimal generator of this group

$$
\lim _{t \rightarrow 0} \frac{1}{t}\left(\mathcal{G}_{n}(-t) f_{n}-f_{n}\right)=-i\left(H_{n} f_{n}-f_{n} H_{n}\right) \doteq-\mathcal{N}_{n} f_{n}
$$

where $H_{n}$ is Hamiltonian (1) and the operator: $-i\left(H_{n} f_{n}-f_{n} H_{n}\right)$ is defined on the domain $\mathcal{D}\left(H_{n}\right) \subset \mathcal{H}_{n}$.

Let us denote by $\{Y\}$ the set consisting of one element $Y \equiv(1, \ldots, s)$. We define the $(1+n)$ th-order $(n \geq 0)$ cumulant of groups of operators (2) as follows

$$
\mathfrak{A}_{1+n}(-t,\{Y\}, X \backslash Y)=\sum_{\mathrm{P}:(\{Y\}, X \backslash Y)=\bigcup_{i} X_{i}}(-1)^{|\mathrm{P}|-1}(|\mathrm{P}|-1) ! \prod_{X_{i} \subset \mathrm{P}} \mathcal{G}_{\left|\theta\left(X_{i}\right)\right|}\left(-t, \theta\left(X_{i}\right)\right),
$$

where $\{Y\}$ is the set consisting of one element $Y=(1, \ldots, s), \sum_{\mathrm{P}}$ is the sum over all possible partitions $\mathrm{P}$ of the set $(\{Y\}, X \backslash Y)=(\{Y\}, s+1, \ldots, s+n)$ into $|\mathrm{P}|$ nonempty mutually disjoint subsets $X_{i} \subset(\{Y\}, X \backslash Y)$ and $\theta:(\{Y\}, X \backslash Y) \rightarrow X$ is the declasterization mapping.

We will consider initial state which is given by the following sequence of marginal density operators

$$
\left.F(t)\right|_{t=0}=\left(1, F_{1}^{0}(1), g_{2}(1,2) F_{1}^{0}(1) F_{1}^{0}(2), \ldots, g_{n}(1, \ldots, n) \prod_{i=1}^{n} F_{1}^{0}(i), \ldots\right),
$$

where the bounded operators $g_{n} \in \mathfrak{L}\left(\mathcal{H}_{n}\right), n \geq 2$, are specified initial correlations. Such initial data is typical for the condensed states of quantum gases, for example, the equilibrium state of 
the Bose condensate satisfies the weakening of correlation condition with the correlations which characterize the condensed state [13]. We remark that nonequilibrium dynamics of correlations was constructed in [14.

The evolution of all possible states is described by the sequence of marginal density operators $F(t)=\left(1, F_{1}(t, 1), \ldots, F_{s}(t, 1, \ldots, s), \ldots\right)$ governed by the initial-value problem of the quantum BBGKY hierarchy

$$
\begin{aligned}
& \frac{d}{d t} F_{s}(t, Y)=\left(\sum_{j=1}^{s}(-\mathcal{N}(j))+\epsilon \sum_{j_{1}<j_{2}=1}^{s}\left(-\mathcal{N}_{\text {int }}\left(j_{1}, j_{2}\right)\right)\right) F_{s}(t, Y)+ \\
& +\epsilon \sum_{j=1}^{s} \operatorname{Tr}_{s+1}\left(-\mathcal{N}_{\text {int }}(j, s+1)\right) F_{s+1}(t, Y, s+1), \\
& \left.F_{s}(t, Y)\right|_{t=0}=g_{s}(Y) \prod_{i=1}^{s} F_{1}^{0}(i), \quad s \geq 1,
\end{aligned}
$$

where on $f_{n} \in \mathfrak{L}_{0}^{1}\left(\mathcal{H}_{n}\right) \subset \mathfrak{L}^{1}\left(\mathcal{H}_{n}\right)$ the operators $(-\mathcal{N}(j))$ and $\left(-\mathcal{N}_{\text {int }}\left(j_{1}, j_{2}\right)\right)$ are defined by the corresponding formula

$$
\begin{aligned}
& (-\mathcal{N}(j)) f_{n} \doteq-i\left(K(j) f_{n}-f_{n} K(j)\right) \\
& \left(-\mathcal{N}_{\text {int }}\right)\left(j_{1}, j_{2}\right) f_{n} \doteq-i\left(\Phi\left(j_{1}, j_{2}\right) f_{n}-f_{n} \Phi\left(j_{1}, j_{2}\right)\right) .
\end{aligned}
$$

If $\left\|F_{1}^{0}\right\|_{\mathfrak{L}^{1}(\mathcal{H})}<e^{-1}$ and $\max _{n \geq 2}\left\|g_{n}\right\|_{\mathfrak{L}\left(\mathcal{H}_{n}\right)}<\infty$, then for $t \in \mathbb{R}$ a nonperturbative solution of the Cauchy problem (5) $-(\sqrt[6]{6})$ is given by the expansion [15]

$$
F_{s}(t, Y)=\sum_{n=0}^{\infty} \frac{1}{n !} \operatorname{Tr}_{s+1, \ldots, s+n} \mathfrak{A}_{1+n}(-t,\{Y\}, X \backslash Y) g_{1+n}(\{Y\}, X \backslash Y) \prod_{i=1}^{s+n} F_{1}^{0}(i)
$$

where $\mathfrak{A}_{1+n}(-t)$ is the $(1+n)$ th-order $(n \geq 0)$ cumulant (41) of groups of operators (2) and $g_{1+n}(\{Y\}, X \backslash Y)$ is the correlation operator of clusters of particles [14], in particular case $n=0$ it has the form: $g_{1+0}(t,\{Y\})=\sum_{\mathrm{P}: Y=\cup_{i} X_{i}} \prod_{X_{i} \subset \mathrm{P}} g_{\left|X_{i}\right|}\left(t, X_{i}\right)$.

In the paper [15] it was proved that, if initial data is completely determined by a one-particle marginal density operator, i.e. $g_{1+n} \equiv I, n \geq 1$, in initial data (6) , then states given in terms of the sequence of marginal density operators (8) can be described within the framework of the sequence $F\left(t \mid F_{1}(t)\right)=\left(1, F_{1}(t), F_{2}\left(t \mid F_{1}(t)\right), \ldots, F_{s}\left(t \mid F_{1}(t)\right), \ldots\right)$ of explicitly defined functionals $F_{s}\left(t \mid F_{1}(t)\right), s \geq 2$, of the solution $F_{1}(t)$ of the generalized quantum kinetic equation.

We extend this statement to case of initial data (6) and construct the mean field (selfconsistent field) asymptotics of a solution of the Cauchy problem of the corresponding generalized quantum kinetic equation.

\section{The generalized quantum kinetic equation in case of correlated initial state}

We reformulate the Cauchy problem (5)-(6) as the new Cauchy problem for a one-particle density operator governed by the generalized quantum kinetic equation and the sequence of 
explicitly defined marginal functionals of the state $F_{s}\left(t, Y \mid F_{1}(t)\right), s \geq 2$, which are determined by the solution $F_{1}(t)$ of such Cauchy problem. With this aim we introduce the following kinetic cluster expansions of cumulants (44) of groups of operators (2)

$$
\begin{aligned}
& \mathfrak{A}_{1+n}(-t,\{Y\}, X \backslash Y) g_{1+n}(\{Y\}, X \backslash Y) \prod_{i=1}^{s+n} \mathfrak{A}_{1}(t, i)= \\
& =\sum_{n_{1}=0}^{n} \frac{n !}{\left(n-n_{1}\right) !} \mathfrak{G}_{1+n-n_{1}}\left(t,\{Y\}, s+1, \ldots, s+n-n_{1}\right) \times \\
& \times \sum_{\mathrm{D}_{\mathrm{s}+\mathrm{n}}: Z=\cup_{i} X_{i}} \sum_{i_{1}<i_{2}<\ldots<i_{\left|\mathrm{D}_{\mathrm{s}+\mathrm{n}}\right|}}^{s+n-n_{1}} \prod_{k=1}^{\left|\mathrm{D}_{\mathrm{s}+\mathrm{n}}\right|} \frac{1}{\left|X_{k}\right| !} \mathfrak{A}_{1+\left|X_{k}\right|}\left(-t, i_{k}, X_{k}\right) \times \\
& \times \prod_{k=1}^{\left|\mathrm{D}_{\mathrm{s}+\mathrm{n}}\right|} g_{1+\left|X_{k}\right|}\left(i_{k}, X_{k}\right) \mathfrak{A}_{1}\left(t, i_{k}\right) \prod_{j \in Z} \mathfrak{A}_{1}(t, j),
\end{aligned}
$$

where $X \backslash Y \equiv(s+1, \ldots, s+n)$ and $\sum_{\mathrm{D}_{\mathrm{s}+\mathrm{n}}: Z=\cup_{i} X_{i}}$ is the sum over all possible dissections $\mathrm{D}_{\mathrm{s}+\mathrm{n}}$ of the linearly ordered set $Z \equiv\left(s+n-n_{1}+1, \ldots, s+n\right)$ on no more than $s+n-n_{1}$ linearly ordered subsets. We give a few examples of recurrence relations (9)

$$
\begin{aligned}
& \breve{\mathfrak{A}}_{1}(t,\{Y\})=\mathfrak{G}_{1}(t,\{Y\}), \\
& \breve{\mathfrak{A}}_{2}(t,\{Y\}, s+1)=\mathfrak{G}_{2}(t,\{Y\}, s+1)+\mathfrak{G}_{1}(t,\{Y\}) \sum_{i_{1}=1}^{s} \breve{\mathfrak{A}}_{2}\left(t, i_{1}, s+1\right), \\
& \breve{\mathfrak{A}}_{3}(t,\{Y\}, s+1, s+2)=\mathfrak{G}_{3}(t,\{Y\}, s+1, s+2)+ \\
& \quad+2 ! \mathfrak{G}_{2}(t,\{Y\}, s+1) \sum_{i_{1}=1}^{s+1} \breve{\mathfrak{A}}_{2}\left(t, i_{1}, s+2\right)+\mathfrak{G}_{1}(t,\{Y\})\left(\sum_{i_{1}=1}^{s} \breve{\mathfrak{A}}_{3}\left(t, i_{1}, s+1, s+2\right)+\right. \\
& \left.\quad+2 ! \sum_{1=i_{1}<i_{2}}^{s} \breve{\mathfrak{A}}_{2}\left(t, i_{1}, s+1\right) \breve{\mathfrak{A}}_{2}\left(t, i_{2}, s+2\right)\right),
\end{aligned}
$$

where the operator $\widehat{\mathfrak{A}}_{1+n}(t)$ is the $(1+n)$ th-order scattering cumulant

$$
\breve{\mathfrak{A}}_{1+n}(t,\{Y\}, X \backslash Y) \doteq \mathfrak{A}_{1+n}(-t,\{Y\}, X \backslash Y) g_{1+n}(\{Y\}, X \backslash Y) \prod_{i=1}^{s+n} \mathfrak{A}_{1}(t, i)
$$

In terms of scattering cumulants (10) the solutions $\mathfrak{G}_{1+n}(t,\{Y\}, X \backslash Y), n \geq 0$, of recurrence relations (9) are represented by the following expansions

$$
\begin{aligned}
& \mathfrak{G}_{1+n}(t,\{Y\}, X \backslash Y) \doteq n ! \sum_{k=0}^{n}(-1)^{k} \sum_{n_{1}=1}^{n} \ldots \sum_{n_{k}=1}^{n-n_{1}-\ldots-n_{k-1}} \frac{1}{\left(n-n_{1}-\ldots-n_{k}\right) !} \times \\
& \times \breve{\mathfrak{A}}_{1+n-n_{1}-\ldots-n_{k}}\left(t,\{Y\}, s+1, \ldots, s+n-n_{1}-\ldots-n_{k}\right) \times \\
& \times \prod_{\substack{\mathrm{D}_{j}: Z_{j}=\bigcup_{l_{j}} X_{l_{j}},\left|\mathrm{D}_{j}\right| \leq s+n-n_{1}-\cdots-n_{j}}}^{k} \frac{1}{\left|\mathrm{D}_{j}\right| !} \sum_{\substack{s+n-n_{1}-\ldots-n_{j} \\
i_{1} \neq \ldots \neq i_{\left|\mathrm{D}_{j}\right|}=1}} \prod_{X_{l_{j}} \subset \mathrm{D}_{j}} \frac{1}{\left|X_{l_{j}}\right| !} \breve{\mathfrak{A}}_{1+\left|X_{l_{j}}\right|}\left(t, i_{l_{j}}, X_{l_{j}}\right),
\end{aligned}
$$


where $\sum_{\mathrm{D}_{j}: Z_{j}=\bigcup_{l_{j}} X_{l_{j}}}$ is the sum over all possible dissections of the linearly ordered set $Z_{j} \equiv$ $\left(s+n-n_{1}-\ldots-n_{j}+1, \ldots, s+n-n_{1}-\ldots-n_{j-1}\right)$ on no more than $s+n-n_{1}-\ldots-n_{j}$ linearly ordered subsets. For example,

$$
\begin{aligned}
& \mathfrak{G}_{1}(t,\{Y\})=\breve{\mathfrak{A}}_{1}(t,\{Y\})=\mathfrak{A}_{1}(-t,\{Y\}) g_{1}(\{Y\}) \prod_{i=1}^{s} \mathfrak{A}_{1}(t, i), \\
& \mathfrak{G}_{2}(t,\{Y\}, s+1)=\breve{\mathfrak{A}}_{2}(t,\{Y\}, s+1)-\breve{\mathfrak{A}}_{1}(t,\{Y\}) \sum_{i=1}^{s} \breve{\mathfrak{A}}_{2}(t, i, s+1), \\
& \mathfrak{G}_{3}(t,\{Y\}, s+1, s+2)=\breve{\mathfrak{A}}_{3}(t,\{Y\}, s+1, s+2)- \\
& \quad-2 ! \breve{\mathfrak{A}}_{2}(t,\{Y\}, s+1) \sum_{i_{1}=1}^{s+1} \breve{\mathfrak{A}}_{2}\left(t, i_{1}, s+2\right)-\breve{\mathfrak{A}}_{1}(t,\{Y\})\left(\sum_{i_{1}=1}^{s} \breve{\mathfrak{A}}_{3}\left(t, i_{1}, s+1, s+2\right)-\right. \\
& \left.\quad-2 ! \sum_{i_{1}=1}^{s} \sum_{i_{2}=1}^{s+1} \widehat{\mathfrak{A}}_{2}\left(t, i_{1}, s+1\right) \breve{\mathfrak{A}}_{2}\left(t, i_{2}, s+2\right)+2 ! \sum_{1=i_{1}<i_{2}}^{s} \breve{\mathfrak{A}}_{2}\left(t, i_{1}, s+1\right) \breve{\mathfrak{A}}_{2}\left(t, i_{2}, s+2\right)\right) .
\end{aligned}
$$

As a result of the application of expansion (9) to solution (8) we establish that the one-particle density operator $F_{1}(t)$ is governed by the following generalized quantum kinetic equation

$$
\begin{aligned}
& \frac{d}{d t} F_{1}(t, 1)=-\mathcal{N}(1) F_{1}(t, 1)+ \\
& +\epsilon \operatorname{Tr}_{2}\left(-\mathcal{N}_{\text {int }}(1,2)\right) \sum_{n=0}^{\infty} \frac{1}{n !} \operatorname{Tr}_{3, \ldots, n+2} \mathfrak{G}_{1+n}(t,\{1,2\}, 3, \ldots, n+2) \prod_{i=1}^{n+2} F_{1}(t, i), \\
& \left.F_{1}(t)\right|_{t=0}=F_{1}^{0} .
\end{aligned}
$$

where the operator $\left(-\mathcal{N}_{\text {int }}(1,2)\right)$ is defined by formula (17), and the $(1+n)$ th-order generated evolution operators $\mathfrak{G}_{1+n}(t), n \geq 0$, are determined by expansion (11) over scattering cumulants (10). In equation (12) the collision integral series converges under the condition that [16]: $\left\|F_{1}(t)\right\|_{\mathfrak{L}^{1}(\mathcal{H})}<e^{-8}$.

The global in time solution of initial-value problem (12)-(13) is determined by the following expansion

$$
F_{1}(t, 1)=\sum_{n=0}^{\infty} \frac{1}{n !} \operatorname{Tr}_{2, \ldots, 1+n} \mathfrak{A}_{1+n}(-t, 1,2, \ldots, n+1) g_{1+n}(1,2, \ldots, n+1) \prod_{i=1}^{n+1} F_{1}^{0}(i),
$$

where $\mathfrak{A}_{1+n}(-t)$ is the $(1+n) t h$-order cumulant (4) of groups of operators (2). The series (14) converges under the condition that [16]: $\left\|F_{1}^{0}\right\|_{\mathfrak{L}^{1}(\mathcal{H})}<e^{-10}\left(1+e^{-9}\right)^{-1}$.

Correspondingly having applied expansion (9) to solutions (8) in case of $s \geq 2$, in terms of solution expansion (14), they are represented as the following marginal functionals of the state

$$
F_{s}\left(t, Y \mid F_{1}(t)\right) \doteq \sum_{n=0}^{\infty} \frac{1}{n !} \operatorname{Tr}_{s+1, \ldots, s+n} \mathfrak{G}_{1+n}(t,\{Y\}, X \backslash Y) \prod_{i=1}^{s+n} F_{1}(t, i),
$$

where the $(1+n)$ th-order generated evolution operator $\mathfrak{G}_{1+n}(t)$ is determined by expansion (11) over scattering cumulants (10). The series (15) converges under the condition that: 
$\left\|F_{1}(t)\right\|_{\mathfrak{L}^{1}(\mathcal{H})}<e^{-(3 s+2)}$. The constructed marginal functionals of the state (15) characterize the correlations of states of quantum many-particle systems.

We note that the average values of the nonadditive-type marginal observables are determined in terms of marginal functionals of the state (15) and in case of the additive-type marginal observables they are determined by solution (14) of the generalized quantum kinetic equation (12).

Thus, in case of initial data (6) solution (8) of the Cauchy problem (5)-(6) of the quantum BBGKY hierarchy and a solution of the Cauchy problem of the generalized kinetic equation (12)-(13) together with marginal functionals of the state (15) give two equivalent approaches to the description of the evolution of quantum many-particle systems. The coefficients of generalized quantum kinetic equation (12) and generated evolution operators (11) of marginal functionals (15) are determined by the operators of initial correlations.

\section{The mean field limit theorems}

In case of initial state involving correlations for an asymptotic perturbation of generated evolution operator (11) in the mean field limit the following equality is valid

$$
\lim _{\epsilon \rightarrow 0}\left\|\mathfrak{G}_{1+n}(t,\{Y\}, X \backslash Y) f_{s+n}\right\|_{\mathfrak{L}^{1}\left(\mathcal{H}_{s+n}\right)}=0, \quad n \geq 1
$$

and in case of the first-order generated evolution operator (11) we have respectively

$$
\lim _{\epsilon \rightarrow 0}\left\|\left(\mathfrak{G}_{1}(t,\{Y\})-\prod_{i_{1}=1}^{s} \mathcal{G}_{1}\left(-t, i_{1}\right) g_{1}(\{Y\}) \prod_{i_{2}=1}^{s} \mathcal{G}_{1}\left(t, i_{2}\right)\right) f_{s}\right\|_{\mathfrak{L}^{1}\left(\mathcal{H}_{s}\right)}=0 .
$$

In view that under the condition that: $t<t_{0} \equiv\left(2\|\Phi\|_{\mathfrak{L}\left(\mathcal{H}_{2}\right)}\left\|\epsilon F_{1}^{0}\right\|_{\mathfrak{L}^{1}(\mathcal{H})}\right)^{-1}$, the series for $\epsilon F_{1}(t)$ converges, then for $t<t_{0}$ the remainders of solution series (14) can be made arbitrary small for sufficient large $n=n_{0}$ independently of $\epsilon$. Then, using stated above asymptotic perturbation formulas for each integer $n$ every term of this series converges term by term to the limit operator $f_{1}(t)$ which is represented in the form of the following expansion

$$
\begin{aligned}
& f_{1}(t, 1)=\sum_{n=0}^{\infty} \int_{0}^{t} d t_{1} \ldots \int_{0}^{t_{n-1}} d t_{n} \operatorname{Tr}_{2, \ldots, 1+\mathrm{n}} \mathcal{G}_{1}\left(-t+t_{1}, 1\right)\left(-\mathcal{N}_{\text {int }}(1,2)\right) \times \\
& \times \prod_{j_{1}=1}^{2} \mathcal{G}_{1}\left(-t_{1}+t_{2}, j_{1}\right) \ldots \prod_{j_{n-1}=1}^{n} \mathcal{G}_{1}\left(-t_{n-1}+t_{n}, j_{n-1}\right) \times \\
& \times \sum_{i_{n}=1}^{n}\left(-\mathcal{N}_{\text {int }}\left(i_{n}, 1+n\right)\right) \prod_{j_{n}=1}^{1+n} \mathcal{G}_{1}\left(-t_{n}, j_{n}\right) g_{1+n}(1, \ldots, n+1) \prod_{i=1}^{1+n} f_{1}^{0}(i)
\end{aligned}
$$

For bounded interaction potentials series (16) is norm convergent on the space $\mathfrak{L}^{1}(\mathcal{H})$ under the condition that: $t<t_{0} \equiv\left(2\|\Phi\|_{\mathfrak{L}\left(\mathcal{H}_{2}\right)}\left\|f_{1}^{0}\right\|_{\mathfrak{L}^{1}(\mathcal{H})}\right)^{-1}$.

Thus, if there exists the limit $f_{1}^{0} \in \mathfrak{L}^{1}(\mathcal{H})$ of initial data (13), namely

$$
\lim _{\epsilon \rightarrow 0}\left\|\epsilon F_{1}^{0}-f_{1}^{0}\right\|_{\mathfrak{L}^{1}(\mathcal{H})}=0
$$


then for finite time interval $t \in\left(-t_{0}, t_{0}\right)$, where $t_{0} \equiv\left(2\|\Phi\|_{\left.\mathfrak{L}_{(\mathcal{H}}\right)}\left\|f_{1}^{0}\right\|_{\mathfrak{L}^{1}(\mathcal{H})}\right)^{-1}$, there exists the mean field limit of solution expansion (14) of the generalized quantum kinetic equation (12):

$$
\lim _{\epsilon \rightarrow 0}\left\|\epsilon F_{1}(t)-f_{1}(t)\right\|_{\mathfrak{L}^{1}(\mathcal{H})}=0
$$

where the operator $f_{1}(t)$ is represented by series (16) and it is a solution of the Cauchy problem of the modified quantum Vlasov kinetic equation

$$
\begin{aligned}
& \frac{d}{d t} f_{1}(t, 1)=-\mathcal{N}(1) f_{1}(t, 1)+ \\
& +\operatorname{Tr}_{2}\left(-\mathcal{N}_{\text {int }}(1,2)\right) \prod_{i_{1}=1}^{2} \mathcal{G}_{1}\left(-t, i_{1}\right) g_{1}(\{1,2\}) \prod_{i_{2}=1}^{2} \mathcal{G}_{1}\left(t, i_{2}\right) f_{1}(t, 1) f_{1}(t, 2), \\
& \left.f_{1}(t)\right|_{t=0}=f_{1}^{0}
\end{aligned}
$$

Since a solution of initial-value problem (12)-(13) of the generalized kinetic equation converges to a solution of initial-value problem (18)-(19) of the modified quantum Vlasov kinetic equation as (17), for marginal functionals of the state (15) we correspondingly establish

$$
\lim _{\epsilon \rightarrow 0}\left\|\epsilon^{s} F_{s}\left(t, Y \mid F_{1}(t)\right)-\prod_{i_{1}=1}^{s} \mathcal{G}_{1}\left(-t, i_{1}\right) g_{1}(\{Y\}) \prod_{i_{2}=1}^{s} \mathcal{G}_{1}\left(t, i_{2}\right) \prod_{j=1}^{s} f_{1}(t, j)\right\|_{\mathfrak{L}^{1}\left(\mathcal{H}_{s}\right)}=0
$$

This equality means the propagation of initial correlations in time in the mean field limit.

\section{On mean field quantum kinetic equations}

Let us consider the pure states, i.e. the operator $f_{1}(t)=\left|\psi_{t}\right\rangle\left\langle\psi_{t}\right|$ is a one-dimensional projector onto a unit vector $\left|\psi_{t}\right\rangle \in \mathcal{H}$ and its kernel has the following form: $f_{1}\left(t, q, q^{\prime}\right)=\psi(t, q) \psi\left(t, q^{\prime}\right)$. Then, in case of initial data, satisfying a chaos property, it holds

$$
\lim _{\epsilon \rightarrow 0} \| \epsilon F_{1}^{0}-\left|\psi_{0}\right\rangle\left\langle\psi_{0}\right| \|_{\mathfrak{L}^{1}(\mathcal{H})}=0
$$

and in this case statement (20) reads

$$
\lim _{\epsilon \rightarrow 0} \| \epsilon^{s} F_{s}\left(t \mid F_{1}(t)\right)-\prod_{i_{1}=1}^{s} \mathcal{G}_{1}\left(-t, i_{1}\right) g_{1}(\{Y\}) \prod_{i_{2}=1}^{s} \mathcal{G}_{1}\left(t, i_{2}\right)\left|\psi_{t}\right\rangle\left\langle\left.\psi_{t}\right|^{\otimes s} \|_{\mathfrak{L}^{1}\left(\mathcal{H}_{s}\right)}=0\right.
$$

where the function $\left|\psi_{t}\right\rangle \in \mathcal{H}$ is a solution of the Cauchy problem of the nonlinear Hartree equation for initial data $\left|\psi_{0}\right\rangle$. In particular, we remark that in case of a system of particles, interacting by the potential which kernel is the Dirac measure, it reduces to the cubic nonlinear Schrödinger equation

$$
i \frac{\partial}{\partial t} \psi(t, q)=-\frac{1}{2} \Delta_{q} \psi(t, q)+|\psi(t, q)|^{2} \psi(t, q)
$$


In case of correlated initial state (6) the sufficient equation for the description of the pure state evolution governed by modified quantum Vlasov kinetic equation (18) is the GrossPitaevskii-type kinetic equation

$$
i \frac{\partial}{\partial t} \psi(t, q)=-\frac{1}{2} \Delta_{q} \psi(t, q)+\int d q^{\prime} d q^{\prime \prime} \mathfrak{b}\left(t, q, q ; q^{\prime}, q^{\prime \prime}\right) \psi\left(t, q^{\prime \prime}\right) \psi^{*}(t, q) \psi\left(t, q^{\prime}\right),
$$

where the coupling ratio function $\mathfrak{b}\left(t, q, q ; q^{\prime}, q^{\prime \prime}\right)$ is the kernel of the scattering length operator: $\prod_{i_{1}=1}^{2} \mathcal{G}_{1}\left(-t, i_{1}\right) b_{1}(\{1,2\}) \prod_{i_{2}=1}^{2} \mathcal{G}_{1}\left(t, i_{2}\right)$.

Observing that in the macroscopic scale of the variation of variables, groups of operators (2) of finitely many particles depend on microscopic time variable $\varepsilon^{-1} t$, where $\varepsilon \geq 0$ is a scale parameter, the dimensionless marginal functionals of the state are represented in the form: $F_{s}\left(\varepsilon^{-1} t \mid F_{1}(t)\right)$. As a result of the limit processing $\varepsilon \rightarrow 0$ we establish the Markovian kinetic evolution (21) with the corresponding coefficient $\mathfrak{b}\left(\varepsilon^{-1} t\right)$.

\section{Conclusion}

We generalized the concept of quantum kinetic equations for the kinetic evolution involving correlations of particle states at initial time [12, for instance, correlations characterizing the condensed states [13]. The mean field scaling asymptotics of a solution of the generalized quantum kinetic equation of many-particle systems in condensed states was analyzed. These results can be extended to quantum systems of bosons or fermions [14].

It should be emphasized that the kinetic evolution is an inherent property of infinite-particle systems. In spite of the fact that in terms of a one-particle marginal density operator from the space of trace-class operators can be described a system with the finite average number of particles, the generalized quantum kinetic equation has been derived on the basis of the formalism of nonequilibrium grand canonical ensemble since its framework is adopted to the description of infinite-particle systems in suitable Banach spaces as well.

We note that one more approach to the construction of the kinetic equations in the mean field limit in case of the presence of correlations at initial time is based on the description of the kinetic evolution in terms of the evolution of marginal observables [17, 18].

We remark that developed approach is also related to the problem of a rigorous derivation of the non-Markovian kinetic-type equations from underlaying many-particle dynamics which make possible to describe the memory effects of particle.

\section{References}

[1] H. Spohn, Kinetic equations from Hamiltonian dynamics: Markovian limits. Rev. Modern Phys. 53, (1980), 569-615.

[2] A. Arnold, Mathematical properties of quantum evolution equations. Lecture Notes in Math. 1946, (2008), 45-110.

[3] R. Adami, F. Golse and A. Teta, Rigorous derivation of the cubic NLS in dimension one. J. Stat. Phys. 127, (6), (2007), 1193-1220. 
[4] C. Bardos, F. Golse, A.D. Gottlieb and N.J. Mauser, Accuracy of the time-dependent Hartree-Fock approximation for uncorrelated initial states. J. Stat. Phys. 115, (2004), $(3 / 4), 1037-1055$.

[5] L. Erdös, B. Schlein and H.-T. Yau, Derivation of the cubic nonlinear Schrödinger equation from quantum dynamics of many-body systems. Invent. Math. 167, (3), (2007), 515-614.

[6] L. Erdös, B. Schlein and H.-T. Yau, Derivation of the Gross-Pitaevskii Equation for the Dynamics of Bose-Einstein Condensate. Ann. of Math., 172, (2010), 291-370.

[7] L. Erdös and B. Schlein, Quantum dynamics with mean field interactions: a new approach. J. Stat. Phys. 134, (5), (2009), 859-870.

[8] M. Grillakis, M. Machedon and D. Margetis, Second-order corrections to mean field evolution of weakly interacting bosons. I. Comm. Math. Phys. 294, (1), (2010), 273301.

[9] A. Michelangeli, Role of scaling limits in the rigorous analysis of Bose-Einstein condensation. J. Math. Phys. 48, (2007), 102102.

[10] L. Saint-Raymond, Kinetic models for superfluids: a review of mathematical results. C. R. Physique, 5, (2004), 6575.

[11] H. Spohn, Kinetic equations for quantum many-particle systems. Preprint arXiv:0706.0807v1, (2007).

[12] C. Cercignani, V.I. Gerasimenko and D.Ya. Petrina, Many-Particle Dynamics and Kinetic Equations. Kluwer, 1997.

[13] M.M. Bogolyubov, Lectures on Quantum Statistics. Problems of Statistical Mechanics of Quantum Systems. Rad. Shkola, 1949 (in Ukrainian).

[14] V.I. Gerasimenko and D.O. Polishchuk, Dynamics of correlations of Bose and Fermi particles. Math. Meth. Appl. Sci. 34, (1), (2011), 76-93.

[15] V.I. Gerasimenko and Zh.A. Tsvir, A description of the evolution of quantum states by means of the kinetic equation. J. Phys. A: Math. Theor. 43, (48), (2010), 485203.

[16] I.V. Gapyak, V.I. Gerasimenko, On rigorous derivation of the Enskog kinetic equation. Preprint arXiv:1107.5572, (2011).

[17] G. Borgioli and V.I. Gerasimenko, Initial-value problem of the quantum dual BBGKY hierarchy, Nuovo Cimento, 33 C, (1), (2010), 71-78.

[18] V.I. Gerasimenko, Heisenberg picture of quantum kinetic evolution in mean-field limit, Kinet. Relat. Models, 4, (1), (2011), 385-399. 